\title{
Analytical calculation of the wake potential of a spherical resonator
}

\author{
Sebastian Ratschow* and Thomas Weiland \\ TEMF, Fachbereich Elektrotechnik und Informationstechnik, Technische Universität Darmstadt, Schlossgartenstrasse 8, \\ D-64289 Darmstadt, Germany
}

(Received 15 March 2002; published 23 May 2002)

\begin{abstract}
An analytical formula for the wake potential of a closed spherical resonator with perfectly conducting walls is presented. Mode analysis is used for the calculation. For every rotationally symmetric TM mode the loss parameter is calculated and the formula for the determination of the corresponding frequency is given. The final wake potential is an infinite sum over all modes mentioned above.
\end{abstract}

DOI: 10.1103/PhysRevSTAB.5.052001

PACS numbers: 41.60.-m, 02.30.Jr, 03.50.De

\section{INTRODUCTION}

In order to test the order of convergence of computer programs for wake field computation with conformal meshing it is desirable to have an analytical formula for a wake potential other than that of the well-known pillbox cavity [1]. The wake potential of a spherical resonator with perfectly conducting walls is well suited for such an evaluation. Still the result presented here can be useful for direct application as well. Superconducting cavities typically tend to look rather like a sphere than like a pillbox.

The resulting formulas for the spherical resonator are simpler to evaluate than the corresponding formulas for the pillbox cavity. This is due to the fact that it takes two parameters (gap width and radius) to specify a pillbox, whereas a sphere is described by its radius alone. In the pillbox, transverse and longitudinal wave numbers are not the same in contrast to the single (radial) wave number in the spherical resonator.

According to [1] where the pillbox cavity is reviewed in detail in the mode analysis approach the wake potential of any closed resonator can be written as an infinite sum

$$
W\left(z_{0}\right)=-2 Q \sum_{\mu} k_{\mu} \cos \left(\omega_{\mu} \frac{z_{0}}{c}\right),
$$

where $\mu$ extends over all relevant modes and $k_{\mu}$ is the loss parameter defined by

$$
k_{\mu}=\frac{V_{\mu} V_{\mu}^{*}}{4 U_{\mu}} .
$$

$U_{\mu}$ is the energy stored in the mode $\mu$, and $V_{\mu}$ is the complex voltage induced by a point charge $Q$ traveling at the speed of light $c$ along the symmetry axis through the cavity under test.

What remains to be done is to find the angular frequencies $\omega_{\mu}$ and the field patterns of the modes and to determine their loss factors. This calculation is carried out for the spherical resonator in the remainder of this paper.

*Electronic address: ratschow@temf.tu-darmstadt.de

\section{CALCULATION OF THE WAKE POTENTIAL}

\section{A. Modes of a spherical resonator}

We start from the equation for the Hertz vector $\Pi_{r}$ in spherical coordinates. We restrict consideration to TM modes without azimuthal $(\varphi)$ dependency as we restrict ourselves to wake potentials for beams passing on the symmetry axis ([2], p. 530):

$$
\frac{\partial^{2} \Pi_{r}}{\partial r^{2}}+\frac{1}{r^{2} \sin \vartheta} \frac{\partial}{\partial \vartheta}\left(\sin \vartheta \frac{\partial \Pi_{r}}{\partial \vartheta}\right)+k^{2} \Pi_{r}=0,
$$

where $k^{2}=\epsilon_{0} \mu_{0} \omega^{2}$. After separation of variables with the ansatz $\Pi_{r}:=R(k r) S(\vartheta), R(k r)$ satisfies

$$
\frac{d^{2} R}{d(k r)^{2}}+\left[1-\frac{n(n+1)}{(k r)^{2}}\right] R=0,
$$

with the well-known solution $R(k r)=\sqrt{k r} Z_{n+1 / 2}(k r)$, where $Z_{n+1 / 2}(k r)$ is a cylinder function of any kind and order $n+1 / 2$, while the equation for $S(\vartheta)$ becomes

$$
\frac{1}{\sin \vartheta} \frac{d}{d \vartheta}\left(\sin \vartheta \frac{d S}{d \vartheta}\right)+n(n+1) S=0,
$$

whose solution is the ordinary (zonal) Legendre polynomial $S(\vartheta)=P_{n}(\cos \vartheta)$ leading to

$$
\Pi_{r}=\sqrt{k r} Z_{n+1 / 2}(k r) P_{n}(\cos \vartheta) e^{i \omega t} .
$$

This solution is valid for all natural $n \geq 1$.

The fields must be finite at the origin and thus we obtain the field components as ([2], p. 626)

$$
\begin{aligned}
& E_{r}(\vartheta, r, t)=k^{2} \frac{n(n+1)}{(k r)^{3 / 2}} J_{n+1 / 2}(k r) P_{n}(\cos \vartheta) e^{i \omega t} \\
& E_{\vartheta}(\vartheta, r, t)= \frac{k}{r} \frac{d}{d(k r)}\left[\sqrt{k r} J_{n+1 / 2}(k r)\right] \\
& \times \frac{d}{d \vartheta} P_{n}(\cos \vartheta) e^{i \omega t} \\
& H_{\varphi}(\vartheta, r, t)=-i \frac{\epsilon_{0} \omega}{r} \sqrt{k r} J_{n+1 / 2}(k r) \\
& \times \frac{d}{d \vartheta} P_{n}(\cos \vartheta) e^{i \omega t}
\end{aligned}
$$


where $J_{n+1 / 2}$ is the Bessel function of the first kind. The tangential electric field at the surface of the sphere vanishes, so the boundary condition to be satisfied reads as

$$
\left.\frac{d}{d(k r)}\left[\sqrt{k r} J_{n+1 / 2}(k r)\right]\right|_{r=R}=0 .
$$

Obviously this equation is equivalent to

$$
\left.\frac{d}{d z} J_{n+1 / 2}(z)\right|_{z=k R}=-\frac{1}{2 k R} J_{n+1 / 2}(k R),
$$

which leads after application of a recursion formula for Bessel functions to the transcendental equation for the determination of the allowed values for $k$ ([2], p. 626)

$$
\frac{J_{n+1 / 2}(k R)}{J_{n-1 / 2}(k R)}=\frac{k R}{n} \text {. }
$$

\section{B. Field energy of the mode}

Because the mean energy contents of the electric and of the magnetic field are the same, we can simply calculate the magnetic energy and double the result

$$
U_{n k}=\frac{\mu_{0}}{2} \int_{V} H_{\varphi} H_{\varphi}^{*} d V .
$$

Specifically we have

$$
\begin{aligned}
U_{n k}= & \pi \epsilon_{0} k^{2} \int_{0}^{R} k r J_{n+1 / 2}^{2}(k r) d r \\
& \times \int_{0}^{\pi}\left[\frac{d}{d \vartheta} P_{n}(\cos \vartheta)\right]^{2} \sin (\vartheta) d \vartheta .
\end{aligned}
$$

The first integral is readily found to be ([2], p. 171)

$$
\int_{0}^{R} k r J_{n+1 / 2}^{2}(k r) d r=\frac{k R^{2}}{2}\left\{\left[\left.\frac{d}{d z} J_{n+1 / 2}(z)\right|_{z=k R}\right]^{2}+\left(1-\frac{\left(n+\frac{1}{2}\right)^{2}}{(k R)^{2}}\right) J_{n+1 / 2}^{2}(k R)\right\} .
$$

By the boundary condition Eq. (11) the integral becomes

$$
\int_{0}^{R} k r J_{n+1 / 2}^{2}(k r) d r=\frac{k}{2}\left(R^{2}-\frac{n(n+1)}{k^{2}}\right) J_{n+1 / 2}^{2}(k R) .
$$

We next turn to the second integral in Eq. (14). Upon substituting $x:=\cos \vartheta$ we find

$$
\int_{0}^{\pi}\left[\frac{d}{d \vartheta} P_{n}(\cos \vartheta)\right]^{2} \sin (\vartheta) d \vartheta=\int_{-1}^{1}\left[\frac{d}{d x} P_{n}(x)\right]^{2}\left(1-x^{2}\right) d x .
$$

When applying the same substitution to Eq. (5) we see that $P_{n}(x)$ satisfies

$$
\frac{d}{d x}\left(\left(1-x^{2}\right) \frac{d P_{n}}{d x}\right)+n(n+1) P_{n}=0 .
$$

With the help of Eq. (18) by partial integration we transform Eq. (17) to

$$
\begin{aligned}
\int_{-1}^{1} \frac{d P_{n}}{d x}\left[\frac{d P_{n}}{d x}\left(1-x^{2}\right)\right] d x & =\left[P_{n} \frac{d P_{n}}{d x}\left(1-x^{2}\right)\right]_{x=-1}^{x=1}-\int_{-1}^{1} P_{n} \frac{d}{d x}\left[\left(1-x^{2}\right) \frac{d P_{n}}{d x}\right] d x \\
& =n(n+1) \int_{-1}^{1} P_{n}^{2} d x=n(n+1) \frac{2}{2 n+1} .
\end{aligned}
$$

(For the last equality see [2], p. 196.)

Finally the value for the energy content of the mode is

$$
U_{n k}=\pi \epsilon_{0} k^{3}\left(R^{2}-\frac{n(n+1)}{k^{2}}\right) \frac{n(n+1)}{2 n+1} J_{n+1 / 2}^{2}(k R) .
$$

\section{Induced voltage of the point charge}

Next we have to calculate the voltage induced by the point charge passing at the speed of light along the symmetry axis through the spherical cavity. This amounts to an integration over the time dependent tangential electric fields on the axis $(\vartheta=0$, respectively, $\vartheta=\pi$ ) (see Fig. 1). Because $E_{r}$ is defined only for $r \geq 0$ the integral has to be carried out in two parts. The first integral will be along the positive $r$ axis $(\vartheta=0)$ and along the positive time axis $(t=r / c)$. The second integral is along the negative time axis $(t=-r / c)$ and along the path where the charge came from $(\vartheta=\pi)$. We chose to set $t=0$ at the center of the cavity for convenience. As only the product $V_{n k} V_{n k}^{*}$ enters the formula for the loss factor, the origin of the time axis is of no importance. Our approach thus reads

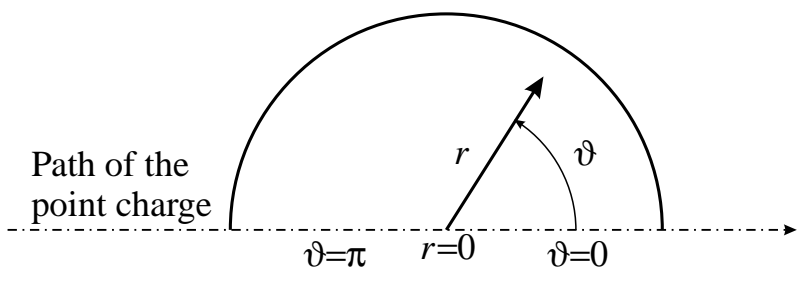

FIG. 1. The coordinate system used in the calculation. 


$$
\begin{aligned}
V_{n k}= & \int_{0}^{R} E_{r}(\vartheta=0, r, t=r / c) d r \\
& +\int_{0}^{R} E_{r}(\vartheta=\pi, r, t=-r / c) d r .
\end{aligned}
$$

Using Eq. (7), taking into account $P_{n}(1)=1, P_{n}(-1)=$ $(-1)^{n}, k=\omega / c$ and with the substitution $z:=k r$ within the integrals we obtain

$$
\begin{aligned}
V_{n k}=k n(n+1)[ & \int_{0}^{k R} \frac{J_{n+1 / 2}(z)}{z^{3 / 2}} e^{i z} d z \\
& \left.+(-1)^{n} \int_{0}^{k R} \frac{J_{n+1 / 2}(z)}{z^{3 / 2}} e^{-i z} d z\right] .
\end{aligned}
$$

Substituting $u_{n}(z):=\sqrt{z} J_{n+1 / 2}(z), \quad v(z):=e^{i z}$, and $w(z):=e^{-i z}$, the above equation reads

$$
\begin{aligned}
V_{n k}= & k\left[\int_{0}^{k R} \frac{n(n+1)}{z^{2}} u_{n} v d z\right. \\
& \left.+(-1)^{n} \int_{0}^{k R} \frac{n(n+1)}{z^{2}} u_{n} w d z\right]
\end{aligned}
$$

$u_{n}(z)$ is a special case of $R(k r)$ above and therefore satisfies Eq. (4)

$$
\frac{d^{2} u_{n}}{d z^{2}}+\left[1-\frac{n(n+1)}{z^{2}}\right] u_{n}=0,
$$

whereas $v(z)$ satisfies

$$
\frac{d^{2} v}{d z^{2}}+v=0
$$

When Eq. (25) is multiplied by $u_{n}$ and subtracted from Eq. (24) multiplied by $v$ one finds in the usual way

$$
\frac{d}{d z}\left[v \frac{d u_{n}}{d z}-u_{n} \frac{d v}{d z}\right]=\frac{n(n+1)}{z^{2}} u_{n} v
$$

The calculation for the second integral goes along the same line. The integrals can now be evaluated to yield

$$
\begin{aligned}
V_{n k}=k\{ & \frac{d u_{n}}{d z}\left[e^{i z}+(-1)^{n} e^{-i z}\right] \\
& \left.-i u_{n}\left[e^{i z}-(-1)^{n} e^{-i z}\right]\right\}_{z=0}^{z=k R} .
\end{aligned}
$$

The exponentials are nonsingular for all values of $z$. The boundary condition Eq. (10) states that $\left.\frac{d u_{n}}{d z}\right|_{z=k R}=0$.
Therefore the first boundary term vanishes at the upper limit. (This is no surprise. The normal derivative of the electric field at a perfectly conducting boundary always vanishes.)

The power series expansion $u_{n}(z)=\sqrt{z} J_{n+1 / 2}(z)$ starts with a term of the order $n+1$ for all $n \geq 1$. This shows that $u_{n}(0)=0$ and $\left.\frac{d u_{n}}{d z}\right|_{z=0}=0$ and leads to

$$
V_{n k}=-i 2 k u_{n}(k R) \begin{cases}\cos (k R) ; & n \text { odd }, \\ i \sin (k R) ; & n \text { even. }\end{cases}
$$

\section{Loss factor for the spherical resonator}

From Eq. (28) we conclude that

$$
V_{n k} V_{n k}^{*}=4 k^{3} R J_{n+1 / 2}^{2}(k R) \begin{cases}\cos ^{2}(k R) ; & n \text { odd, } \\ \sin ^{2}(k R) ; & n \text { even. }\end{cases}
$$

Now we are ready to calculate the loss factor according to Eq. (2). From Eqs. (20) and (29) we obtain

$$
k_{n k}=\frac{R(2 n+1)}{\pi \epsilon_{0}\left(R^{2}-\frac{n(n+1)}{k^{2}}\right) n(n+1)} \begin{cases}\cos ^{2}(k R) ; & n \text { odd, } \\ \sin ^{2}(k R) ; & n \text { even. }\end{cases}
$$

This solution is inserted into Eq. (1) to yield the desired wake potential. The formula for the determination of the resonant frequencies was given already in Eq. (12).

Note that unlike the solution for the pillbox cavity as given in [1] this wake potential contains no Bessel functions. This was meant, when it was claimed, that the result is easier to evaluate than that of the pillbox cavity.

\section{SUMMARY}

Although numerical methods have become the standard approach for computing wake potentials, there is still a need for analytical solutions for serving as reference and for validation. Former results for pillbox cavities are not well suited for convergence tests for boundaries not parallel to the coordinate axes in cylindrical coordinates. In this paper we derived an analytical expression for the wake potential of a spherical resonator which is well suited for validation of computer codes applied to curved structures.

This work was supported in part by DESY, Hamburg, Germany.

[1] T. Weiland and B. Zotter, Part Accel. 11, 143-151 (1981).

[2] K. Simonyi, Theoretische Elektrotechnik (VEB Deutscher Verlag der Wissenschaften, Berlin, 1956). 